\title{
Redefining Dialysis Facility Recommendations for Infection Prevention and Control during the Covid-19 Outbreak
}

\author{
Issa Kawalit ${ }^{1 *}$, Muhand Eltwal ${ }^{2}$, Abbas Elkhatib ${ }^{3}$, Ala Sh. Ali ${ }^{4}$, Dmytro D Ivanov ${ }^{5}$, Wiam Hussein ${ }^{6}$, \\ Walid Aboujaoude ${ }^{7}$, Abdulhafid Shebani ${ }^{8}$, Rania Derani ${ }^{9}$ and Laith Al-Rabadi ${ }^{10}$
}

${ }^{1}$ Chief Medical Officer, Arab Renal Care Group, Amman Jordan

${ }^{2}$ Chief Medical Officer, New England Sini Hospital, MA, USA

${ }^{3}$ Mercer Medical Group of Nephrology, Princeton, WV, USA

${ }^{4}$ Department of Nephrology, Medical City, Baghdad, Iraq

${ }^{5}$ Department of Nephrology, Shupyk National Medical Academy of Postgraduate Education, Kiev, Ukraine

${ }^{6}$ Department of Endocrinology, Royal Bahrain Hospital, Bahrain

${ }^{7}$ Department of Nephrology, Middle East Institute of Health, Bsalim, Lebanon

${ }^{8}$ Department of Nephrology, Tripoli University Hospital, Tripoli, Libya

${ }^{9}$ Kidney Surgical Hospital, Ministry of Health, Damascus, Syria

${ }^{10}$ Renal Division, University of Utah Hospital, UT, USA

${ }^{*}$ Corresponding author: Issa Kawalit, Chief Medical Officer, Arab Renal Care Group, Amman, Jordan, Tel: +962777741008; E-mail: ikawalit@yahoo.com

Received date: April 18, 2020; Accepted date: April 22, 2020; Published date: April 29, 2020

Citation: Kawalit I, Eltwal M, Elkhatib A, Ali AS, Ivanov DD, et al. (2020) Redefining Dialysis Facility Recommendations for Infection Prevention and Control during the Covid-19 Outbreak. Arch Med Vol.12 Iss.2:7

Copyright: (C2020 Kawalit I, et al. This is an open-access article distributed under the terms of the Creative Commons Attribution License, which permits unrestricted use, distribution, and reproduction in any medium, provided the original author and source are credited.

\section{Abstract}

In December 2019, pneumonia cases of unknown aetiology were reported in Wuhan, Hubei Province, People's Republic of China (PRC). Its clinical symptoms are similar to those of viral pneumonia. After analysis on respiratory samples, PRC Centers for Disease Control (CDC) experts declared that pneumonia was caused by a highly contagious novel coronavirus (SARS-CoV-2.) The World Health Organization (WHO) has declared the ongoing outbreak as a global public health emergency. Furthermore, advised, in the absence of any apparent pharmaceutical intervention, that the only strategy against (SARS-CoV-2) is to reduce the mixing of susceptible and infectious people through early ascertainment of cases or reduction of contact. Over the last month, the number of cases has risen rapidly in South Korea, Japan, Europe, and the United States. New cases are being diagnosed in the Middle East region. This is even more critical for hemodialysis patients who need maintenance hemodialysis sessions thrice-weekly, which are routinely done in close proximity to other patients in the small space of hemodialysis units. Herein, we report the opinion of a group of nephrologists with the hope that this will serve as a guideline to prepare the nephrology community to deal with this impending danger.
Keywords: Hemodialysis; COVID-19

\section{Introduction}

Novel Coronavirus disease (COVID-19) is a newly reported infectious disease caused by severe acute respiratory syndrome (SARS)-coronavirus (CoV)-2 virus. It primarily manifests as an acute respiratory illness with alveolar and interstitial pneumonia, but it can affect multiple organs such as the heart, kidney, digestive tract, and nervous system [1]. The infection was first identified in 2019 in Wuhan, China, and has since spread globally, resulting in the 2019-20 coronavirus pandemic [2]. As of April 10, 2020, 1,619,051 cases of COVID-19 have been reported worldwide in more than 200 countries, with 96,966 deaths [3]. The infection typically spreads from one person to another via respiratory droplets produced during coughing and sneezing [4,5]. Time from exposure to onset of symptoms is generally between 2 and 14 days, with an average of 5 days $[6,7]$. The standard method of testing is reverse transcription-polymerase chain reaction ( $r R T$ $\mathrm{PCR}$ ) from a nasopharyngeal swab. The viral infection can also be diagnosed from a constellation of symptoms, risk factors, and a chest CT scan showing features of pneumonia $[8,9]$. SARS-CoV-2 has been identified as a bat-origin CoV. The full genome sequence of the COVID-19 virus shows a close resemblance with the bat SARS-like coronavirus strain BatCov RaTG13 that belongs to the Beta coronavirus genus [10]. 


\section{Literature Review}

Previous coronavirus infections, SARS-CoV and the Middle East Respiratory Syndrome Coronavirus (MERS-Co-V), have infected more than 10,000 people in the past two decades, with mortality rates of $10 \%$ and $37 \%$, respectively $[11,12]$.

The infection has been reported in all ages, including children. The majority of symptoms are mild, presenting with a flu-like illness. The common symptoms of COVID-19 are fever (98\%), cough (76\%), myalgia and fatigue (18\% each) with accompanying leucopenia (25\%), and lymphopenia (63\%) [13]. Clinical presentations of upper respiratory infection with rhinorrhea and productive cough are uncommon, except in children. About $16 \%$ to $20 \%$ of cases have been classified as critical. Out of the 41 patients described by Huang et al. [14] all had pneumonia with abnormal findings on chest computerized tomographic examination, and 32\% required management at the critical care unit. High plasma levels of cytokine (interleukin [IL]-2, IL-7, IL-10, monocyte chemoattractant protein 1 , granulocyte-colony stimulating factor, interferon-inducing protein-10, macrophage inflammatory protein-1a were present in patients requiring critical care unit admission [15]. Severe cytokine storms were reported in severe cases and have been associated with increased mortality. Therefore, the treatment of cytokine storm has become an essential component of managing severe patients. Interleukin-6 (IL-6) plays a vital role in Cytokine Release Syndrome (CRS). Hence IL- 6 blockers a potential role in treating severe inflammatory status. Some reports suggest that severe complications are uncommon in children [16].

While social distancing will help minimize the spread of COVID-19, the practice is near impossible for thousands of vulnerable patients with End-Stage Renal Disease (ESRD) who can potentially become severely ill without their regular dialysis. Receiving maintenance dialysis is crucial and lifesaving for ESRD patients. Measures of self-isolation and staying away from other patients is not an option. Hence the need to come up with new measures tailored to our patient population. ESRD patients dialyze next to 10 to 30 other immunocompromised patients for 3 to 4 hours, three times a week in the limited space of the dialysis unit. Unfortunately, there is no easy alternative. While other patients may be able to put off scheduled doctor's appointments to avoid infection, dialysis cannot be postponed.

Kidney involvement associated with COVID-19 seems to be frequent in this infection, with Acute Kidney Injury (AKI) being an independent predictor of mortality. Although the impact of COVID-19 on chronic kidney disease has not yet been reported, COVID-19 infection presents a special threat to diabetic patients on dialysis [17]. In a recent report from Wuhan University, there were 7184 patients on hemodialysis (HD) in 61 dialysis facilities in Wuhan City. At a single HD facility in Renmin Hospital at Wuhan University, 37 out of 230 patients on HD and 4 out of 33 staff members developed the COVID-19 infection between January 14 and February 17, 2020. A total of 7 patients on HD died who had the COVID-19 infection. However, the deaths were attributed to cardiovascular causes and not directly to the COVID-19 infection [18].

Interestingly, patients on HD with COVID-19 had less lymphopenia and lower serum levels of inflammatory cytokines. This should not imply that patients with chronic kidney disease or dialysis-dependence do better and are at lower risk of infection. Management of patients on hemodialysis who had suspected exposure to COVID-19 should be carried out according to strict protocols to minimize the risk to other patients and healthcare personnel taking care of these patients. Although the recommended measures to prevent infection include frequent hand washing, maintaining distance from others (social distancing), and keeping hands away from the face, the use of masks is recommended only for those suspected to have the virus and their caregivers, but not for the general public $[19,20]$.

Herein we present the perspective of a group of nephrologists who felt the need for urgent intervention and more strict measures for patients with ESRD on regular hemodialysis at dialysis centers.

Literature is still scarce in terms of the management of HD patients with COVID-19. We have looked at different guidelines and protocols, including the Chinese Society of Nephrology and the Italian Society of Nephrology, who have had the highest number of COVID-19 cases [21-24]. They managed to develop guidelines for hemodialysis patients during the COVID-19 outbreak. We tried through this review to assemble the most crucial guidelines and expert opinions and tailor them to our patient population. We hope that the review provided below would serve as a guideline in the management of COVID-19 patients requiring hemodialysis.

\section{General Recommendations}

- Personnel carrying the virus (ascertained) must avoid contact with patients on dialysis until cleared by the infectious disease specialist [21-26].

- A team consisting of dialysis physicians, nursing staff, and technologists should receive training in updated clinical practice and management of COVID-19, performing nasopharyngeal swabs for PCR COVID-19 with adequate protection, assessing infection risk, utilizing epidemic prevention tools, and acquiring guidelines from the government, academic societies, and hospital authorities and delivering them to all medical care personnel as needed, preferably online.

- Information on travel, occupation, contacts, and cluster history of each medical staff, dialysis patient, their family members, residents of the same household, and colleagues at work should be reported and updated regularly.

- Group activities, including group studies, group rounds, and case discussions, should be minimized and ideally performed through E-learning.

- Nursing staff must self-monitor their health and immediately inform the director and their supervisor if they have developed symptoms suggestive of COVID-19 infection or came in contact with the COVID-19 case. 
- Activate a triage procedure before the patients enter the dialysis room by taking contact history with COVID-19 infected person and history of fever or respiratory symptoms.

- Suspected cases of COVID-19 infection should be treated as positive COVID-19 until cleared by an infectious disease specialist.

- Reduce the time in the waiting areas and regulate the flow in the dedicated locker rooms.

- Staff and patients must measure body temperature before entering the dialysis facility.

- All patients must wear a face mask during their trip to reach the dialysis facility, in the waiting room, and during the hemodialysis session.

- We advise all patients to wear chlorine dioxide deodorant badge during the journey to reach the dialysis facility, in the waiting room and during the hemodialysis session [27-30].

- Facilities should have space in waiting areas for patients to sit separated from other patients by at least 6 feet. Medically stable patients can wait in a vehicle or outside the dialysis facility where they can be reached by phone when it is their turn to receive treatment.

- We recommend limiting the number of caregivers to the minimum and, if possible, prohibit their presence in the dialysis unit.

- If a patient has undergone the hemodialysis treatment in the dialysis facility, and later tested positive for COVID-19, the areas used must be made available to other patients only after adequate sanitization. Moreover, we would recommend tracing their contacts and implementing quarantine measures according to the adopted policies.

- It is recommended that dialysis staff have meals at different times to avoid eating together. Masks, goggles, and hats should be removed before meals, and hands washed with flowing water and soap. Talking during meals should be avoided to reduce the spread of droplets.

- Patients and accompanying persons can bring small snacks such as candy to prevent hypoglycemia.

- Family members living with patients on hemodialysis must follow the precautions given to patients to prevent personto-person transmission of COVID-19, which includes good personal hygiene and prompt reporting of potentially suspected case of COVID-19.

- Patients who have family members or caregiver subject to quarantine can have hemodialysis as usual after their COVID-19 test is negative and was cleared by an infectious disease specialist to continue regular dialysis.

- Once the family members of a patient become infected with COVID-19, the dialysis patient should be treated as a patient with suspected COVID-19 infection, as noted above.

\section{Patient-specific disinfection recommendations}

- The patient should be sanitized before the arrival at the dialysis facility by encouraging them to wash hands using hand hygiene with alcohol solution Patient encouraged to scrub the sole of their shoes against a mat filled with chlorine dioxide /or use disposable shoe cap.
- The patient should wear disposable medical caps, face masks, gloves, and disposable protective suits. It is essential to wear and remove Personal Protective Equipment (PPE) in the correct order to reduce cross-contamination. Removed PPE should be placed in the assigned medical waste bags as infectious waste.

\section{Staff specific disinfection recommendations}

- Staff should perform hand and face hygiene.

- The staff should wear disposable medical head caps, surgical masks, goggles, gloves, and disposable protective suits. It is vital to wear or remove PPE in the correct order, which can help reduce cross-contamination. Removed PPE should be placed in the assigned medical waste bags as infectious waste.

- Staff should carefully discard their mask each time they exit the isolation station and put a new mask when entering.

\section{Dialysis Facility specific disinfection recommendations}

- Ventilation systems for dialysis facility settings should be designed and modified by engineers in collaboration with infection-control and occupational health specialists [30-32].

- The dialysis facility should enhance the management of airconditioning systems and natural ventilation according to the facility structure, layout, and local climate conditions. The air circulation should be strengthened by taking practical measures, like opening the door and windows when the temperature is appropriate and when the air-conditioning system is used.

- For air disinfection, dialysis facility should.

- Use natural ventilation and strengthen air conditioning ventilation.

- Disinfect and clean the exhaust fan once a month or as recommended by occupational health staff and infectioncontrol specialists.

- The dialysis facility should take measures to classify waste, collect used masks, and remove them in time. Garbage containers should be disinfected with chlorine-containing disinfectants by spraying or wiping regularly.

- The dialysis facility should frequently disinfect the contacted object surfaces in general population areas (check-in counters, verification credential counters, elevator buttons, handrails, etc.), by spraying and wiping with $250 \sim 500 \mathrm{mg} / \mathrm{L}$ chlorine-containing disinfectant or $250 \mathrm{mg} / \mathrm{L}$ chlorine dioxide or as recommended by occupational health personal and infection-control specialist.

- The dialysis facility should continue to follow the infection control requirements related to cleaning and disinfection using products with Environmental Protection Agencyapproved emerging viral pathogens claims recommended for use against COVID-19.

- The dialysis facility disinfection should be performed by professionals twice daily, before starting the hemodialysis sessions and at the end of the hemodialysis sessions. They should disinfect the air and environment integrated by using the vaporization (gasification) hydrogen peroxide disinfection device or with chlorine dioxide. 
- The doors and windows should be closed before disinfection and opened for ventilation after 60 minutes. After disinfection, they should wipe the surface of all objects according to the dialysis facility disinfection protocol.

- The contaminated key areas should be sprayed or wiped with chlorine-containing disinfectant for 60 minutes.

- The dialysis facility staff should clean the machines with disinfectants according to the manufacturer's instructions and as recommended by the occupational health personal and infection-control specialist.

- The dialysis facility staff should disinfect the hemodialysis system according to the manufacturer instructions and as recommended by the occupational health personal and infection-control specialist.

- The dialysis facility staff should ensure that equipment is in good working condition and placed in the correct storage areas.

\section{Suspected or confirmed COVID-19 infection recommendations}

- Clinically stable patients with suspected or confirmed COVID-19 infection should be admitted to a negativepressure isolation room in the dialysis facility. If the capacity of the dialysis facility is overloaded, a designated dialysis facility or hospital should be assigned for treating COVID 19 positive patients [22-25,33].

- An unstable dialysis patient should be dialyzed in the critical care unit in a negative pressure isolation room.

- Consider lowering the frequency of dialysis sessions (2/ week) for patients with stable clinical status and good urine output and provided it is safe for the patient.

- In addition to the patient hand hygiene and wearing PPE, a surgical mask is preferred in this situation.

- The dialysis machines and the equipment that may come into contact with patients or with potentially contaminated material should be disinfected according to standard protocols, as mentioned above.

- If a newly confirmed case of novel coronavirus infection in a dialysis facility is identified, the disinfection procedures should be carried out immediately. Areas in close contact with these infected patients should not be used until cleared by infection control specialist.

- The medical waste from confirmed patients with novel coronavirus infection should be considered as infectious medical waste and disposed of accordingly.

- Patients who need surgery should be screened for the novel coronavirus before the surgery except if it is an urgent procedure.

- The surgical procedure should be done in the patient's inpatient negative pressure room.

- Intubation \& extubating should occur in a negative pressure room using a filter on the Vent circuit.

The dialysis facility with overloaded isolation capacity without the availability of nearby designated dialysis facility for a patient with COVID-19, have two options that are not strongly recommended and are considered as a temporary measure until the medical authority identifies a dialysis facility for patients with COVID-19:

\section{The fixed dialysis care model}

- Patients will continue HD at the original HD facility without changing to another facility.

- Avoid changing dialysis shifts and caregiver staff to avoid cross-contamination and infection.

- Patients with respiratory symptoms should be brought to an appropriate treatment zone as soon as possible to decrease the time in the waiting room.

- Facilities should maintain 6 feet of separation between symptomatic masked patients and other patients during a hemodialysis session.

- Public transport should not be used, and patients should use personal transportation. Transport personnel should wear surgical or $\mathrm{N} 95$ masks throughout. Also, the transport vehicle should be disinfected after each trip.

- Hemodialysis session should start at the last shift of the day until the infection is excluded.

- Avoid sharing the pick-up and drop-off points with other dialysis patients. Avoid entering and exiting with other patients at the same time.

- The route, mode, and time of the transport of dialysis personnel should be fixed. The masked patient should be treated at the end-of-row station or the corner, far from the main traffic flow, if an isolated room is not available. Maintain at least 6 feet separation of at least 6 feet between the masked patient and other patients during treatment.

\section{Hepatitis B isolation room}

Hepatitis B isolation rooms can be used to dialyze patients if the patient with suspected or confirmed COVID-19 is hepatitis $B$ surface antigen-positive or the facility has no hepatitis $B$ surface antigen-positive patients that require treatment in the isolation room. The room should be disinfected after each treatment, as noted above.

\section{Points to remember}

- The protective equipment should be worn in the sequence of the disposable medical cap, surgical mask, protective suits, goggles, and medical rubber gloves. After staff duty, the protective equipment should be removed in the sequence of gloves, hand disinfection, goggles, protective suits, hand disinfection, masks, and caps. Please be aware of the following points:

- Disinfect hand before wearing protective equipment.

- Replace face masks as recommended by the manufacturer and by the infection-control specialists.

- Thoroughly cover all hair and hairline shreds by wearing a cap.

- Immediately replace protective equipment when it contacts the passenger's blood, vomit, and other pollutants with infection risk.

- Disinfect and dry reusable goggles after each use.

- Carefully take off protective equipment without touching the face with both hands when.

- Discard the disposable protective equipment into the assigned medical waste bag. 
- The shoe soles should be disinfected by footpads containing disinfectant.

\section{Discussion on Controversies}

There is a shortage of ventilators to care for patients infected with COVID-19, and this may require the terrifying prospect of rationing resources. Some sites have developed guidelines for this situation, based on the Sequential Organ Failure Assessment (SOFA) score, which correlates with immediate mortality (patients with high scores and a low likelihood of survival would have to be taken off ventilators to make way for patients more likely to benefit). The SOFA score includes serum creatinine, and it was initially intended to be based on a change from baseline (AKI). It is not clear how to score patients with ESRD, who would start with a high SOFA score if their serum creatinine concentration pre-dialysis were used in calculating the value. Clear data about outcomes in COVID-19 infected patients on chronic hemodialysis is not available; if their prognosis is not severely affected, using their pre-dialysis serum creatinine would unfairly label them as unsalvageable.

\section{Future Solutions}

In an era when just every medical diagnosis and treatment that can be done at home is moving in that direction, only $12 \%$ of patients receiving kidney dialysis in the United States of America get their treatment at home, despite being cheaper and just as safe especially with the availability of online technology. Home dialysis will potentially help lower the rate of transmitted infection [34]. Another modality that should be encouraged is the Peritoneal Dialysis (PD), which is the most cost-effective dialysis and as efficacious compared to HD [35]. Kidney transplantations have led to substantial cost savings for many health care systems. An increase in kidneys transplantation has the potential to reduce the cost of renal replacement therapy further and lower any potential spread of infection compared to hemodialysis at dialysis units [36]. However, this should be cautiously decided separately at every transplant center with weighing the risks and benefits based on the prevalence of COVID-19 in that region.

\section{Conclusion}

Some centers opted to hold living donations for the time being but continued to perform deceased kidney transplants. The concern stems from uncertainty regarding the risk of infection in those recently transplanted patients with their higher risk of acquiring opportunistic infections. On the other hand, patients approaching the need for dialysis with potential living donors represent a clinical dilemma that mandates careful assessment taking into consideration the risk of infection versus the cardiovascular risk entailed with the cumulative time on dialysis. This will raise more questions than answers, and future studies and data would hopefully help address these issues.

\section{Disclaimer}

The views presented in this paper represent the views of the authors and not of the organizations, they represent.

\section{References}

1. Wang D, Hu B, Hu C (2020) Clinical Characteristics of 138 Hospitalized Patients With 2019 Novel Coronavirus-Infected Pneumonia in Wuhan, China. JAMA. 323: 1061-1069.

2. https: //www.who.int/docs/default-source/coronaviruse/ situation-reports/20200307-sitrep-47-covid-19.pdf? sfvrsn=27c364a4_4

3. https: //www.who.int/emergencies/diseases/novelcoronavirus-2019/situation-reports

4. https: //web.archive.org/web/20150124020044/http: www.metapathogen.com/

5. Polgreen PM, Chen Z, Segre AM, Harris ML, Pentella MA, et al. (2009) Optimizing influenza sentinel surveillance at the state level. Am J Epidemiol. 170: 1300-1306.

6. Ginsberg J, Mohebbi MH, Patel RS, Brammer L, Smolinski MS, et al. (2008) Detecting influenza epidemics using search engine query data. Nature. 457: 1012-1014.

7. Chan EH, Sahai V, Conrad C, Brownstein JS (2011) Using web search query data to monitor dengue epidemics: a new model for neglected tropical disease surveillance. PLoS Negl Trop Dis. 5: e1206.

8. Siettos $\mathrm{Cl}$, Russo L (2013) Mathematical modeling of infectious disease dynamics. Virulence. 4: 295-306.

9. Wesolowski A, Metcalf CJ, Eagle N, Kombich J, Grenfell BT, et al. (2015) Quantifying seasonal population fluxes driving rubella transmission dynamics using mobile phone data. Proc Natl Acad Sci USA. 112: 11114-11119.

10. https: //www.who.int/docs/default-source/coronaviruse/whochina-joint-mission-on-covid-19-final-report.pdf

11. https: //www.who.int/csr/sars/country/table2004_04_21/en/

12. http: //www.who.int/emergencies/mers-cov/en/

13. https: //web.archive.org/web/20100107002810/http: www.doh.wa.gov/notify/other/glossary.htm

14. Huang C, Wang Y, Li X, Ren L, Zhao J, et al. (2020) Clinical features of patients infected with 2019 novel coronavirus in Wuhan, China. The Lancet. 395: 497-506.

15. Zhang C, Wu Z, Li JW, Zhao H, Wang GQ (2020) The cytokine release syndrome (CRS) of severe COVID-19 and Interleukin-6 receptor (IL-6R) antagonist Tocilizumab may be the key to reduce the mortality. Int J Antimicrob Agents.

16. https: //www.cdc.gov/coronavirus/2019-ncov/specific-groups/ children-faq.html

17. Naicker S, Yang CW, Hwang SJ, Liu BC, Chen JH, et al. (2020) The Novel Coronavirus 2019 epidemic and kidneys. Kidney Int 2538: 30251-30259.

18. Ma Y, Diao B, Lv X, Zhu J, Liang W, et al. (2020) 2019 Novel coronavirus disease in hemodialysis (HD) patients: Report from one HD center in Wuhan, China. medRxiv.

19. Ikizler TA, Kliger AS (2020) Minimizing the risk of COVID-19 among patients on dialysis. Nat Rev Nephrol 2:1. 
20. Kaltenpoth M, Engl T (2013) Defensive microbial symbionts in Hymenoptera. Defensive Symbiosis. 28: 315-327.

21. Expert Team of Chinese Medical Association Nephrology Branch (2020) Recommendations for prevention and control of novel coronavirus infection in blood purification center (room) from Chinese Medical Association Nephrology Branch. Chin J Nephrol. 36: 82-84.

22. Hwang SJ (2020) Guideline for dialysis facilities during COVID-19 outbreak. Taiwan Society of Nephrology.

23. https: //www.era-edta.org/en/wp-content/uploads/2020/03/ Recommendations-for-Dialysis.COVID-19-SIN-ER.pdf

24. https: //www.era-edta.org/en/wp-content/uploads/2020/03/ COVID_guidelines_finale_eng-GB.pdf

25. https: //www.cdc.gov/coronavirus/2019-ncov/infectioncontrol/ infection-prevention-control-faq.html

26. CDC guidance for dialysis safety including infection prevention tools: https: //www.cdc.gov/dialysis/index.html

27. Deodorant Sanitization with Chlorine Dioxide power for prevention of respiratory infection in Diabetic patients. Abstract, 2nd Bahrain Diabetes and Endocrine Review Conference, which was held on February 20-21,2020 in Bahrain.

28. Deodorant Sanitization with Chlorine Dioxide power for prevention of respiratory infection post living Kidney transplant. Abstract, 3rd Joint Meeting of the Turkish Transplantation Society and the Turkic World Transplantation Society, which was held on October 10-11, 2019 in the capital of Uzbekistan.
29. Ogata N, Shibata T (2009) Effect of chlorine dioxide gas of extremely low concentration on absenteeism of school children. Int J Med Med Sci. 1: 288-289.

30. Miura T, Shibata T (2010) Antiviral Effect of Chlorine Dioxide against Influenza Virus and Its Application for Infection Control. The Open Antimicrobial Agents Journal. 2: 71-78.

31. Lowe JJ, Gibbs SG, Iwen PC, Smith PW, Hewlett AL (2013) Impact of Chlorine Dioxide Gas Sterilization on Nosocomial Organism Viability in a Hospital Room. Int J Environ Res Public Health. 10: 2596-2605.

32. https: //www.ecdc.europa.eu/sites/default/files/documents/ RRA-sixth-update-Outbreak-of-novel-coronavirus-disease-2019COVID-19.pdf

33. https: //www.cdc.gov/coronavirus/2019-ncov/healthcarefacilities/dialysis.html

34. Hager D, Ferguson TW, Komenda P (2019) Cost Controversies of a "Home Dialysis First" Policy. Can J Kidney Health Dis. 6.

35. Pike E, Hamidi V (2017) More Use of Peritoneal Dialysis Gives Significant Savings: A Systematic Review and Health Economic Decision Model. J Clin Med Res. 9: 104-116.

36. Jarl J, Desatnik P (2018) Do kidney transplantations save money? A study using a before-after design and multiple register-based data from Sweden. Clin Kidney J. 11: 283-288. 